\title{
THE ‘BIRTH-AND-ASSASSINATION’ PROCESS
}

\author{
David ALDOUS * \\ The University of California, Berkeley, CA, USA \\ William B. KREBS \\ Florida State University, Talahassee, FL, USA
}

Received January 1989

Revised September 1989

\begin{abstract}
A new variant of a branching process is introduced, with sufficient conditions for it to persist and to die out. The model is applied to discuss the asymptotic stability of a new type of queuing process.
\end{abstract}

Keywords: Branching processes, multi-server queues.

Consider a system of particles evolving according to the following rules:

(a) Particles $j$ have random (i.i.d.) lifetimes $K_{j}$;

(b) During its lifetime, a particle has offspring at the times of a Poisson (rate $\lambda$ ) process.

This is a well-studied type of branching process. The populations in successive generations behave as the simple Galton-Walton branching process with mean offspring equal to $\lambda E K$, and so the process is subcritical or supercritical according as this mean is less than or greater than 1 .

We study a variation in which the 'clock' which counts down time until a particle's death does not start ticking until the particle's parent dies. Thus each particle has an initial period of time (until its parent's death) in which it may have offspring but is not at risk of dying; after its parent's death, it lives a further random time $K$, during which it may have further offspring. While the particle lives, its offspring may have their own offspring, and so forth, and none of its descendants are at risk of dying. In other words a typical configuration of the process is a finite collection of 'clans', and only the current heads of clans can be killed (hence the term 'assassination').

We wish to find conditions under which all particles are eventually removed from the system and to note a connection between this particle system and a queuing model for parallel processing.

Formally, let $N$ denote the positive integers and let $N^{\mathrm{f}}=\bigcup_{n=0}^{\infty} N^{n}$ be the set of finite $n$-tuples of positive integers (with $N^{0}=\emptyset$ ). For $n \in N^{\mathrm{f}}$, let $k(\boldsymbol{n})$ be the number of coordinates in $\boldsymbol{n}$, and let $\boldsymbol{\sigma}(\boldsymbol{n})=n_{1}+\cdots+n_{k(\boldsymbol{n})}$. Let $k(\emptyset)=0=\boldsymbol{\sigma}(\emptyset)$.

Let $\left\{X_{n}\right\}, n \in N^{f}$, be a family of independent Poisson processes with common arrival rate $\lambda$. Let $\left\{K_{n}\right\}, n \in N^{\mathrm{f}}$, be a family of independent, strictly positive random variables, with common distribution function $F$. Suppose the families $\left\{X_{n}\right\}$ and $\left\{K_{n}\right\}$ are independent.

The particle system starts at time 0 with only the ancestor particle, indexed by $\varnothing$. This particle produces offspring at the arrival times of $X_{\varnothing}$, which enter the system with indices (1), (2), . according to their birth order. Each new particle $n$ entering the system immediately begins producing offspring at the arrival times of $X_{n}$; the offspring of $n$ are indexed $(n, 1),(n, 2), \ldots$ also according to birth order.

* Research supported by NSF Grant MCS87-01426. 
The ancestor particle is 'at risk' at time 0 . It continues to produce offspring until time $Y_{\not \varnothing}=K_{\not \varnothing}$, when it is removed from the system. Let $k>0$ and let $\boldsymbol{n}=\left(n_{1}, \ldots, n_{k-1}, n_{k}\right), \boldsymbol{n}^{\prime}=\left(n_{1}, \ldots, n_{k-1}\right)$. When a particle $\boldsymbol{n}^{\prime}$ is removed from the system (at time $Y_{n^{\prime}}$ ), $\boldsymbol{n}$ then becomes 'at risk'; it continues to produce offspring until time $Y_{n}=Y_{n^{\prime}}+K_{n^{\prime}}$, when it is removed from the system.

We will say that the birth-and-assassination process is stable if with probability 1 there exists some time $t<\infty$ when no particles remain in the system. We will say that the process is unstable if it is not stable.

Theorem. Let $\mathscr{B}$ be a birth and assassination process with offspring rate $\lambda$ whose killing distribution has moment generating function $\phi$. Suppose $\phi$ is finite in some neighborhood of 0 . If $\min _{u>0} \lambda u^{-1} \phi(u)<1$ then the process is stable. If $\min _{u>0} \lambda u^{-1} \phi(u)>1$ then the process is unstable.

Remark. Such a natural model and result may have applications in various areas in which branching process models are used. Our particular motivation was Tsitsiklis, Papadimitreou and Humblet (1986), in which a queuing system with blocking was introduced to model a data base that prevents two queries from modifying the same entry simultaneously. In this system, the arrival times of jobs are a Poisson process with rate $\lambda$, the service times are independent and have distribution $F$, and there are infinitely many servers. Suppose job $j_{n+1}$ arrives while jobs $j_{1}, \ldots, j_{n}$ are receiving or awaiting service; then for $i=1, \ldots, n$, there is a fixed probability $p, 0<p<1$, that service for $j_{n+1}$ cannot begin until service for $j_{i}$ is completed, and the events $\left\{j_{n+1}\right.$ is blocked by $\left.j_{i}\right\}, i=1, \ldots, n$, are mutually independent.

Say that $j_{n}$ is a descendant of $j_{m}$ if $j_{n}$ is blocked by $j_{m}$ or is a descendant of a job blocked by $j_{m}$, and let $Y_{n}$ denote the particle system of descendants of $j_{n}$. If $\lambda \rightarrow \infty$ and $p \rightarrow 0$ so that $\lambda p \rightarrow C$ with $0<C<\infty$, it is not hard to show that $Y_{n}$ converges in distribution to a birth-and-assassination process with arrival rate $C$ and residual lifespan distribution $F$. Here, convergence of processes means, as usual, convergence on bounded time intervals. Rigorously relating the asymptotic behaviors is a more challenging problem: it seems intuitively clear that the stability threshold for the Tsitsiklis-Papadinitreou process should converge to the stability threshold for the birth and assassination process given in our theorem.

Proof of Theorem. Let $\left\{B_{\nu 1}\right\}, \nu, i=1,2, \ldots$, be independent exponential random variables with parameter $\lambda$. Let $\left\{K_{i}\right\}$ be independent random variables with distribution function $F$ and moment generating function $\phi(u)$.

Certainly, the process $\mathscr{B}$ is stable if the expected total number of offspring is finite.

$$
E[\text { number of particles created }]=\sum_{n} P[n \text { is born }] .
$$

For the particle $\left(n_{1}, \ldots, n_{k}\right)$ to be born, its ancestor in generation $i$ must have had at least $n_{i}$ offspring for each $i$ less than $k$. Thus,

$$
P[\boldsymbol{n} \text { is born }]=P\left[\sum_{i=1}^{j} \sum_{\nu=1}^{n_{i}} B_{\nu i}<\sum_{i=1}^{j} K_{i}, j=1, \ldots, k(\boldsymbol{n})\right] \leqslant P\left[\sum_{i=1}^{k(n)} \sum_{\nu=1}^{n_{i}} B_{\nu i}<\sum_{i=1}^{k(n)} K_{i}\right] .
$$

Simple computations show

$$
\begin{aligned}
\sum_{n} P\left[\sum_{i=1}^{k(n)} \sum_{\nu=1}^{n_{i}} B_{v i}<\sum_{i=1}^{k(n)} K_{i}\right] & \leqslant \sum_{n} E\left[\exp u\left(\sum_{i=1}^{k(n)} K_{i}-\sum_{i=1}^{k(n)} \sum_{\nu=1}^{n_{i}} B_{v i}\right)\right](u>0) \\
& =\sum_{n} \prod_{i=1}^{k(n)} E\left[\exp \left(u K_{i}\right)\right] \prod_{i=1}^{k(n)} \sum_{\nu=1}^{n_{1}} E\left[\exp \left(-u B_{v i}\right)\right] \\
& =\sum_{k=1}^{\infty} \phi(u)^{k} \sum_{k(n)=k}(\lambda /(\lambda+u))^{\sigma(n)} .
\end{aligned}
$$


The second and third equations in the preceding sequence follow from the independence of $\left\{K_{i}\right\}$ and $\left\{B_{v i}\right\}$ and from properties of exponential distributions, respectively. We now calculate

$$
\sum_{k(n)=k}\left(\frac{\lambda}{\lambda+u}\right)^{\sigma(n)}=\left(\frac{\lambda}{u}\right)^{k} \sum_{m=k}^{\infty}\left(\begin{array}{c}
m-1 \\
k-1
\end{array}\right)\left(\frac{u}{\lambda+u}\right)^{k}\left(\frac{\lambda}{\lambda+u}\right)^{m-k}=\left(\frac{\lambda}{u}\right)^{k} .
$$

So,

$$
E[\text { number of particles created }] \leqslant \sum_{k=1}^{\infty}\left(\phi(u) \frac{\lambda}{u}\right)^{k} .
$$

If $\min _{u>0} \lambda u^{-1} \phi(u)<1$, then the expected number of particles created is finite. This proves the first part of the theorem.

To prove the second part of the theorem, we use a large deviation estimate for $P[\boldsymbol{n}$ is born]. The following result and argument are well-known, but we cannot supply a citation, so we sketch the proof.

Lemma 1. Let $X_{1}, X_{2}, \ldots$, be i.i.d. random variables with $E X<0$ and $P[X>0]>0$. Let $E \mathrm{e}^{u X}=\psi(u)$ be finite in some neighborhood of 0 , and let $\rho=\min _{u>0} \psi(u)$. Then

$$
\lim _{n \rightarrow \infty} n^{-1} \log P\left[\sum_{1}^{k} X_{j}>0, k=1, \ldots, n\right]=\log \rho .
$$

Sketch of Proof. Chernoff's theorem for large deviations gives this limit for $n^{-1} \log P\left[\sum_{1}^{n} X,>0\right]$. Conditioning on $\sum_{1}^{n} X_{i}$ and considering cyclic shifts of the $X_{i}^{\prime}$ 's gives

$$
1>P\left[\sum_{1}^{k} X_{j}>0, k=1, \ldots, n\right] / P\left[\sum_{1}^{n} X_{j}>0\right]>n^{-1} \text {. }
$$

Lemma 2. If $\min _{u>0} \lambda u^{-1} \phi(u)>1$, then there exists $k$ such that $\sum_{k(n)=k} P[\boldsymbol{n}$ is born $]>1$.

Proof. Let $N_{1}, N_{2}, \ldots$, be i.i.d. shifted geometric random variables, with $P[N=j]=p q^{j-1}, j=1,2, \ldots$, where $p>0, q-1-p$, and let $N_{1}, N_{2}, \ldots,\left\{K_{j}\right\}$ and $\left\{B_{j p}\right\}$ be mutually independent. For $n=1,2, \ldots$, define $Z_{n}=K_{n}-\sum_{\nu=1}^{N_{n}} B_{n \nu} \cdot\left\{Z_{n}\right\}$ is a sequence of i.i.d. random variables. An easy calculation shows that $E \mathrm{e}^{u Z}=\phi(u) \lambda p /(\lambda p+u)$. Conditioning on $\left(N_{1}, \ldots, N_{k}\right)=\left(n_{1}, \ldots, n_{k}\right)$ gives

$$
P\left[\sum_{1}^{j} Z_{i}>0, j=1, \ldots, k \mid\left(N_{1}, \ldots, N_{k}\right)=\left(n_{1}, \ldots, n_{k}\right)\right]=P\left[\sum_{i=1}^{j} \sum_{\nu=1}^{n_{i}} B_{\nu i}>\sum_{i=1}^{j} K_{i}, j-1, \ldots, k\right] .
$$

Multiply by $P\left[\left(N_{1}, \ldots, N_{k}\right)=\left(n_{1}, \ldots, n_{k}\right)\right]$ and sum over $\boldsymbol{n}$ such that $k(\boldsymbol{n})=k$, giving

$$
\begin{aligned}
P\left[\sum_{1}^{j} Z_{i}>0, j=1, \ldots, k\right] & =\sum_{k(n)=k} P\left[\sum_{i=1}^{j} \sum_{\nu=1}^{n_{i}} B_{\nu i}>\sum_{i=1}^{j} K_{i}, j=1, \ldots, k\right] p^{k} q^{n_{1}+\cdots+n_{k}} \\
& =p^{k} \sum_{k(n)=k} P[\boldsymbol{n} \text { is born }] q^{\sigma(n)} .
\end{aligned}
$$


If $\min _{u>0} \lambda u^{-1} \phi(u)=1+\varepsilon$, choose $p$ sufficiently small that $p^{-1} \min _{u>0} E \mathrm{e}^{u Z}=\min _{u>0} \phi(u) \lambda /\left(\lambda_{p}+\right.$ $u)>1+\frac{1}{2} \varepsilon$. By applying Lemma 1 to the sequence $\left\{Z_{n}\right\}$, we see that for $k$ sufficiently large,

$$
p^{k} \sum_{k(n)=k} P[n \text { is born }] q^{\sigma(n)}=P\left[\sum_{1}^{j} Z_{i}>0, j=1, \ldots, k\right]>p^{k}\left(1+\frac{1}{2} \varepsilon\right)^{k} .
$$

Divide by $p^{k}$ and let $p \rightarrow 0$. The Lebesgue monotone convergence theorem gives

$$
\sum_{k(n)=k} P[n \text { is born }]>\left(1+\frac{1}{2} \varepsilon\right)^{k}>1 .
$$

Proof of Theorem (continued). Say that a particle in the process is special if it is the ancestor particle, of if it is a particle in generation $n k$ that is descended from a special particle in generation $(n-1) k$ with no living ancestors. The expected number of special particles in generation $n k$ is greater than $\left(1+\frac{1}{2} \varepsilon\right)^{n k}$, so the process of special particles is a supercritical branching process.

With positive probability, this process never terminates, implying that there is a positive probability that the birth-and-assassination process' main process never terminates.

\section{Acknowledgement}

We wish to thank the referee for his helpful comments and for suggesting an improvement in the statement of the main theorem.

\section{References}

Tsitsiklis, J., C. Papadimitreou and P. Humblet (1986), The performance of a precedence based queueing discipline, $J$. Assoc. of Comput. Mach. 33(3), 593-602. 